\title{
La cientificidad de las ciencias de la información: fundamentación filosófica 0 resolución de problemas
}

\author{
Pablo Melogno *
}

Artículo recibido:

29 de septiembre de 2011.

Artículo aceptado:

23 de febrero de 2012.

\section{RESUMEN}

Se presenta una discusión acerca de las condiciones de cientificidad de las ciencias de la información a partir de las ideas de Thomas Kuhn. Se realiza una revisión de las diferentes acepciones del concepto de paradigma, y de las diferencias señaladas por Kuhn entre las ciencias naturales y las ciencias sociales. Se revisa la perspectiva kuhniana de que las diferencias entre ambos tipos de disciplinas están dadas por la ausencia de debates acerca de los fundamentos y la naturaleza disciplinar en las ciencias naturales, y la ausencia de tradiciones consolidadas de resolución de problemas en las ciencias sociales. En función de esto, se busca establecer que las estrategias de fundamentación filosófica

* Universidad de la República, Uruguay. pmelogno@gmail.com

INVESTIGACIÓN BIBLIOTECOLÓGICA, Vol. 26, Núm. 56, enero/abril, 2012, México, ISSN: 0187-358X. pp. 13-29 
de una disciplina no son definitorias para determinar su carácter científico, en cuanto la condición fundamental para la consolidación paradigmática radica en la conformación de una tradición exitosa de resolución de problemas, y en la asunción de elementos metodológicos y teóricos que no se someten a cuestionamiento.

Palabras clave: Kuhn, Epistemología, Ciencias de la información.

\section{ABSTRACT}

\section{The Scientificity of Information Sciences:} Philosophical Foundation or Problem Solving Pablo Melogno

The paper presents a discussion on the conditions of scientificity of information sciences, based on Thomas Kuhn's ideas. It also conducts a review of the different meanings of the concept of paradigm, and the differences noted by Kuhn between natural sciences and social sciences. Additionally, it examines the Kuhnian perspective which states that the differences between both types of disciplines involve mainly the absence of debate about the foundations and the disciplinary nature in natural sciences, and the absence of consolidated problem-solving traditions in social sciences. On this basis, the paper intends to establish that the philosophical foundation strategies of a certain discipline are not a defining criterion to determine its scientific nature, since the fundamental condition for the paradigmatic consolidation lies in the formation of a successful problem-solving tradition, and the adoption of methodological and theoretical elements that do not undergo questioning.

Keywords: Kuhn, Epistemology, Information Sciences.

\section{INTRODUCCIÓN}


tal status una vez que cumple con determinadas características provenientes de un criterio de cientificidad, o de una definición de ciencia. Parece ser igualmente frecuente en el debate la idea de que la fundamentación filosófica o epistemológica de una disciplina juega un papel importante en la legitimación de sus pretensiones de cientificidad. En este debate ha ejercido una notoria influencia la figura y la obra de Thomas Kuhn, y aunque ésta fue originalmente concebida para las ciencias naturales, ha sido aplicada de las formas más variadas al estudio de las disciplinas sociales. ${ }^{1}$ Las ciencias de la información ${ }^{2}$ no han estado exentas de los debates involucrados en la cientificidad de los estudios sociales, ni tampoco de las alternativas que el pensamiento de Kuhn ofrece para la clarificación de su status científico. El propósito de este trabajo es ofrecer un análisis desde la perspectiva kuhniana de algunos aspectos relativos a las condiciones de cientificidad de las ciencias de la información, en el contexto de las ciencias sociales, y más específicamente, del papel que la fundamentación especulativa y la resolución de problemas juegan en la consolidación científica de una disciplina. En este sentido no se discutirá si está justificada la inclusión de las ciencias de la información en las ciencias sociales, ${ }^{3}$ o si las pretensiones de cientificidad de las disciplinas que componen las ciencias de la información son o no legítimas. Se partirá de la base de que las ciencias de la información poseen pretensiones de cientificidad, y que estas pretensiones son legítimas pero problemáticas, en tanto que las condiciones bajo las que las disciplinas pertenecientes a las ciencias de la información pueden ser declaradas como científicas permanecen aún sujetas a debate. ${ }^{4}$

En función de esto se intentará establecer que las estrategias de fundamentación filosófica de una disciplina no son definitorias para establecer su carácter científico, y que más bien la condición fundamental para la cientificidad de una disciplina radica en la conformación de una tradición exitosa de resolución de problemas, y en la asunción de elementos metodológicos y

1 Algunos trabajos clásicos en la literatura anglosajona son los de Friedrichs (1970) Eckberg y Hill (1980), Ritzer (1980), Barnes (1982), y Fuller (2000). En la literatura en español pueden mencionarse Gómez Rodríguez (1997), Velazco Gómez (2000), Otero (2004) y Cordero Ulate, (2008).

2 A lo largo del texto se utilizarán de modo indistinto términos como bibliotecología y ciencias de la información, por no ser objeto de este trabajo los debates respecto a las diferencias y relaciones entre estos y otros términos afines. Remitimos para ello a los trabajos de de Souza (1996) y Linares (2004).

3 Para una discusión de este punto puede consultarse Morales López (1998) Ávila Araújo (2003).

4 Lo que se quiere señalar aquí es que si bien la expresión "ciencias de la información" es de uso corriente en la literatura, aún permanecen sujetas a debate las razones por las que se puede, o no, justificar su empleo. 
teóricos que, en cuanto han resultado exitosos, no se someten a cuestionamiento. ${ }^{5}$ De este modo, se buscará defender que el tratamiento de las pretensiones de cientificidad de las ciencias de la información remite antes a la sistematización y ampliación de los instrumentos de resolución de problemas específicos del campo, que a la fundamentación filosófica de las bases conceptuales de la disciplina.

\section{FundAMENTACIÓN DISCIPLINAR}

¿Qué implica fundamentar una disciplina? ¿Qué tipo de procedimiento conceptual supone el esclarecer los fundamentos de una teoría? Expresiones como fundamentar una disciplina, clarificar las bases conceptuales de una disciplina, o definir su naturaleza, no parecen referirse en su uso habitual a los éxitos empíricos logrados por quienes cultivan la disciplina, o por los descubrimientos que han realizado o los problemas que han resuelto. Si hablamos de fundamentar la teoría de la relatividad intuitivamente no nos remitimos a enlistar los descubrimientos hechos por la física de Einstein, lo mismo que si hablamos de fundamentar el psicoanálisis no nos referiremos a presentar las técnicas y descubrimientos clínicos dados a partir de Freud.

En los términos en que usualmente se concibe, el ejercicio de fundamentación de una teoría no remite al trabajo de ajustar de la teoría con el dominio de fenómenos que le son específicos, ni a los problemas que ésta logra resolver en este dominio, sino a un trabajo de tipo especulativo desarrollado mediante el análisis de la estructura conceptual de la teoría. Si hablamos de fundamentar la física de Einstein podemos estar hablando de ofrecer razones para aceptar la noción de espacio derivada de la relatividad, del mismo modo que si hablamos de fundamentar el psicoanálisis podemos intentar clarificar la noción freudiana de pulsión o la imagen de la naturaleza humana propuesta por Freud. Este trabajo presenta manifestaciones diferentes en

5 La noción de tradición científica, así como la importancia de la resolución de problemas para la consolidación disciplinar, han sido tratadas por Larry Laudan. Para Laudan, la evaluación de una teoría remite a determinar si ésta resuelve problemas relevantes en su dominio, más que a determinar si cumple con algún criterio como la verdad, la corroboración o la justificación (1977: 41). Asimismo, consideró las tradiciones de investigación como una serie de supuestos de amplio alcance acerca de los objetos que componen el dominio de una disciplina, y de los métodos más adecuados para resolver los problemas de dicho dominio. De forma similar a los programas de investigación de Lakatos (1970), las tradiciones de investigación poseen una función metodológica que contiene indicaciones sobre lo que está permitido y lo que está vedado dentro del trabajo científico. A esto se suma una función ontológica, que se vincula con la definición de los objetos de estudio que dentro de la tradición se entienden como legítimos (idem: 116 y ss.). Agradezco a los evaluadores anónimos de la revista por ésta y otras valiosas sugerencias. 
función de la disciplina y del tipo de estrategia de fundamentación que se tome, pero puede reducirse en principio a dos variantes. Una primera estrategia puede consistir en asumir un criterio de cientificidad o una definición de ciencia, y en detectar en la teoría en cuestión las características que certifican su cumplimiento del criterio, y por lo tanto su cientificidad. Una segunda estrategia puede consistir en asociar o deducir una teoría científica o con pretensiones de cientificidad, a un sistema filosófico especulativo, ubicando en el sistema filosófico los fundamentos conceptuales últimos de la teoría que se pretende fundamentar. Una tercera estrategia ${ }^{6}$ puede consistir en analizar una disciplina científica y a partir del análisis identificar sus fundamentos, ya sean éstos filosóficos, teóricos, metodológicos o de otro orden. Esta última estrategia no es incompatible con las anteriores al no parecer haber razones para atribuirle el término fundamentación en mayor o menor medida que a las dos primeras. ${ }^{7}$

En este marco han sido múltiples los intentos por definir a la bibliotecología o la ciencia de la información, bajo la premisa de que una definición y una caracterización precisa de su campo de trabajo resultan determinantes para el desarrollo de la disciplina. Es de referencia en este sentido ${ }^{8}$ la publicación, en 1972 de The Foundations of Education For Librarianship, donde J. Shera emprende metódicamente esta actividad de fundamentación de la ciencia bibliotecológica, a efectos de ubicarla dentro de las ciencias sociales y de esclarecer sus bases y presupuestos epistemológicos, (Shera, 1980). Asimismo Rendón Rojas (2000) ofrece una revisión de los intentos de definir la disciplina desde finales de la década de los años sesenta hasta los noventa, refiriendo a clásicos como R. Taylor (1963), Isakovic (1965), Borko (1968), y López Yepes (1978). Más recientemente, también pueden mencionarse los trabajos de Ávila Araújo (2003) y Capurro (2007).

Frente a este campo de debate y tratamiento de las bases teóricas de las ciencias de la información, una revisión del concepto kuhniano de paradigma puede servir para clarificar en qué nivel se sitúa el trabajo de fundamentación involucrado en estas estrategias. En La estructura de las revoluciones

6 Indicada por una evaluador anónimo.

7 Esto en cuanto las tres estrategias pueden ser identificadas con correlativa frecuencia en la literatura. Cuando Popper (1934) declara la cientificidad de la física relativista y la no cientificidad del psicoanálisis con base en el criterio falsacionista de demarcación, está apelando a la primera estrategia. Cuando Gadamer (1965) propone la hermenéutica como marco de fundamentación filosófica de las ciencias humanas, está utilizando la segunda; cuando Heisenberg (1971) ubica el platonismo en la base de los presupuestos filosóficos de la mecánica cuántica, está recurriendo a la tercera estrategia.

8 Se listan a continuación, de forma inevitablemente parcial, algunos abordajes cuya mención se entiende pertinente en cuanto que representan intentos definidos de ofrecer alguna clase de fundamentación del carácter científico de las ciencias de la información. 
cientificas (ERC), Kuhn distingue cuatro elementos constitutivos de los paradigmas: en primer término lo que denomina generalizaciones simbólicas, las que contienen las expresiones formales o formalizables acerca del dominio de estudio del paradigma, y que les proporcionan a los científicos la base de un lenguaje compartido; en segundo término aparecen las bases metafísicas del paradigma, compromisos de nivel general que cumplen la función de delimitar el mundo compartido por los miembros de la comunidad, las metáforas predominantes y los problemas cuya resolución es significativa; en tercer término incluye los valores, una de cuyas funciones es regular las elecciones de los científicos entre alternativas de trabajo incompatibles, y que con cierto nivel de variabilidad remiten a la exactitud de las predicciones, la capacidad de resolver problemas y la coherencia. Por último aparecen los ejemplares paradigmáticos; esto es, casos de problemas que han sido resueltos con éxito por la comunidad, y que estructuran el contenido de la educación científica (Kuhn, 1962: 280-286).

La distinción que hace Kuhn de los diferentes sentidos de paradigma es relevante para una conceptualización epistemológica de las ciencias de la información en varios sentidos. Primero advierte sobre la polisemia que ha arrastrado el concepto desde la primera edición de La estructura de las revoluciones científicas, polisemia de la que el mismo Kuhn se ha reconocido parcial responsable, (Kuhn, 1974: 317-319). Segundo, muestra que un paradigma contiene elementos que si bien están relacionados, no dejan de ser significativamente heterogéneos; y como consecuencia de esto, permite entrever que al hablar de paradigmas en cualquier campo, ya sea de ciencias sociales o naturales, es preciso especificar a cuál de los componentes se está haciendo referencia. Esto previendo que expresiones como "paradigmas en ciencias de la información" o "paradigma bibliotecológico" pueden hacer referencia a un conjunto de compromisos ontológicos, a una serie de problemas resueltos con éxito, o a otro de los componentes de un paradigma (pueden incluso hacer referencia a más de uno, y en algún caso a todos), pero el alcance y las implicaciones que se presentarían en cada caso serían diferentes, por lo que una asunción no explicitada del significado de paradigma resultaría por demás problemática.

Buscando explicitar este tipo de diferencias, Rendón Rojas identifica cada uno de los componentes introducidos por Kuhn con aspectos específicos de las ciencias de la información. Primero remite al nivel de las generalizaciones simbólicas la dificultad de darle forma a una terminología común de la comunidad bibliotecológica, y en los compromisos ontológicos ubica la identificación de la "sociedad del conocimiento" como fenómeno a ser estudiado por las ciencias de la información. En cuanto a los valores, menciona los problemas suscitados en torno a la metodología de la disciplina, y, por último, en 
cuanto a los ejemplares identifica los debates y las dificultades para lograr un modelo satisfactorio de enseñanza de la bibliotecología, (Rendón Rojas, 2000: 38-39).

El planteo de Rendón Rojas señala elementos de las ciencias de la información correspondientes a cada uno de los niveles paradigmáticos de Kuhn, pero también pueden identificarse abordajes que se sitúan definidamente en el nivel de lo que Kuhn denominó bases metafísicas del paradigma. De Souza (1996: 14) denomina problema epistemológico a la indagación vinculada con

los estudios teóricos y filosóficos, los conceptos, la terminología explícita sobre la ciencia en particular; comprende también los estudios de su interdisciplinariedad y metodología, y principalmente, el estudio de su objeto.

Morales López (1998: 38-39) por su parte, sostiene una metodología bibliotecológica, entendida como un estudio filosófico de las herramientas metodológicas presentes en la disciplina. Finalmente Capurro (2007) propone una investigación epistemológica de las ciencias de la información. Este tipo de abordajes se sitúan a nivel de la elucidación de los supuestos filosóficos del paradigma, bajo la premisa de que este trabajo de fundamentación filosófica o especulativa sobre la naturaleza de la disciplina contribuye a la consolidación disciplinar o la legitimación de sus pretensiones de cientificidad. Sin embargo, puede pensarse que la obra de Kuhn proporciona elementos para establecer que este tipo de estrategias no constituye una condición para consolidar el status científico de una disciplina, ya que dicho status se obtiene fundamentalmente en los procesos de aparición de ejemplares paradigmáticos, esto es, cuando las comunidades logran darle forma a procedimientos exitosos para la resolución de problemas.

\section{Consenso y MADUREZ Disciplinar}

En La estructura de las revoluciones cientificas, Kuhn ofrece algunas sugestiones interesantes a modo de contraste entre las ciencias naturales y las sociales. En las ciencias naturales los debates sobre los fundamentos disciplinares son mucho menos frecuentes que en las ciencias sociales, pero no porque los científicos naturales tengan respuestas definidas sobre los fundamentos de los métodos que utilizan o la pertinencia de los problemas que tratan de resolver, sino porque sus comunidades se delimitan en torno a realizaciones metodológicas y teóricas que son universalmente reconocidas y aceptadas sin discusión, (Kuhn, 1962: 13). 
Más adelante, insiste en que los debates acerca de la cientificidad de las disciplinas sociales son análogos a los que existían en las ciencias naturales en los períodos preparadigmáticos. Una de las motivaciones de estos debates remite a que los progresos observables en las ciencias naturales son mucho mayores que en las sociales, lo que lleva a quienes tratan estas cuestiones en las ciencias sociales a aislar en éstas las características que permiten declarar su cientificidad, o a proponer definiciones de ciencia que se apliquen a la disciplina cuya cientificidad se pretende demostrar. A este respecto, Ávila Araújo (2003: 21-22) señala que desde la década de los años sesenta del siglo XX en adelante, el debate en las ciencias de la información ha estado centrado en esclarecer el origen de la disciplina, su definición y sus fundamentos teóricos. Sander (1989: 34) por contrapartida, menciona al planteo de Busha y Harter (1980) como un intento de delimitación de la bibliotecología con base en la consolidación de métodos y herramientas específicas de resolución de problemas. Frente a estas dos alternativas divergentes, cabe pensar que desde una matriz kuhniana las estrategias de fundamentación y definición no dan cuenta de las razones que separan las disciplinas científicas maduras de las que no lo son, en cuanto que esta diferencia está dada por el acuerdo respecto a los problemas relevantes y las herramientas adecuadas para resolverlos.

Ritzer (1975) señaló tempranamente que al aplicar los conceptos kuhnianos a su campo, los sociólogos confundían el nivel de los conceptos metafísicos implícitos que soportan la práctica científica con el nivel disciplinar vinculado a la resolución de problemas; Friedrichs (1979), por ejemplo, identificaba un paradigma funcionalista y un paradigma dialéctico como marcos conceptuales del trabajo sociológico. En términos similares, Puntel Mostafa (1996: 18-19) identifica en la bibliotecología un paradigma cuántico con referencia en las ciencias naturales, y por otro lado los paradigmas de las ciencias sociales, entre los que ubica al positivismo, el marxismo y el estructuralismo, no obstante también dentro de las ciencias sociales denomina paradigmas al liberalismo norteamericano, la social-democracia alemana y el pos-estructuralismo francés, (idem: 20). Ávila Araújo (2003: 24-26) mientras tanto, señala que en sus orígenes las ciencias de la información se sitúan en un modelo funcionalista y positivista, para luego pasar a un modelo marxista, y desembocar finalmente en un modelo de ciencia post-moderna. Capurro introduce diez paradigmas epistemológicos que han influido en el desarrollo histórico de la ciencia de la información.'

Frente a esto, Kuhn enfatizó que si en algunas disciplinas como la economía los debates sobre el método, la cientificidad y los fundamentos son

9 Capurro (2007) identifica los siguientes paradigmas: 1. hermenéutica, 2. racionalismo crítico, 3. teoría crítica, 4. semiótica, 5. constructivismo, 6. cibernética de segundo orden, 7. teoría de sistemas, 8. paradigma físico, 9. paradigma cognitivo, 10. paradigma social. 
menos frecuentes que en otras campos de las ciencias sociales, no es porque los economistas tengan mejores definiciones de ciencia o criterios de cientificidad más sólidos, sino porque las características de la investigación que se desarrolla en su campo hace innecesario este tipo de debate. En el pasado teorías como la física de Aristóteles, la astronomía geocéntrica o la química del flogisto alcanzaron status paradigmáticos gracias a que resolvieron con éxito durante cierto tiempo problemas específicos de su campo; y del mismo modo, fueron abandonadas cuando dieron con problemas que resultaron irresolubles. La consolidación del campo se obtiene cuando la comunidad científica logra un nivel de consenso lo suficientemente alto como para reconocer una serie de logros pasados, construyendo una tradición de investigación con base en dichos logros y en las herramientas que permitieron obtenerlos, (idem: 248). Eso hace que a medida que se produce la consolidación de una disciplina, ciertas zonas del trabajo teórico empiezan a funcionar como compromisos consensuados por la comunidad, y quedan fuera del debate. Refiriéndose a un foro desarrollado en 1996 durante el XIV Coloquio de Bibliotecología, dice Rendón Rojas (1996, 34)

entre los participantes no surgió el cuestionamiento de que si la Bibliotecología era o no una disciplina teórica. Se dio por hecho que es una ciencia, con una historia relativamente corta, pero unida a una práctica muy antigua...

Este tipo de acuerdos parece ser históricamente el resultado de la madurez que se alcanza al menos en algunos sectores de la disciplina. Sin embargo, es necesario especificar que el consenso comunitario y la confianza incondicional en determinados elementos teóricos y metodológicos no es resultado de una convención dada al interior de la comunidad, sino de que estos elementos demostraron un alto nivel de eficacia en la resolución de problemas específicos del campo. Esto llevó a Kuhn a advertir a las comunidades de científicos sociales (en las que su obra ha resultado particularmente influyente) que no es posible construir un paradigma por decreto:

Si... algunos practicantes de las ciencias sociales toman de mí el punto de vista de que pueden mejorar el status de su campo, legislando primero un acuerdo respecto a los puntos fundamentales para después dedicarse a la resolución de rompecabezas, están reconstruyendo mal mi posición (Kuhn, 1970: 168).

La negativa de Kuhn a caracterizar de esta forma la construcción del consenso comunitario responde a que la madurez disciplinar requiere que la comunidad haya logrado al menos un mínimo nivel de progreso con base en las 
herramientas que le dan contenido al consenso, por lo que el logro de un alto grado de consenso comunitario no es una condición metodológica para la consolidación disciplinar, sino una consecuencia de la resolución exitosa de uno o más problemas propios del campo. Es cierto igualmente que una vez consolidado, el consenso comunitario se organiza en torno a los supuestos metafísicos del paradigma, pero las bases históricas del consenso se sitúan en los problemas que la comunidad ha logrado resolver con éxito, y no en los supuestos metafísicos que ulteriormente ha aceptado.

Es relevante en este sentido discriminar el requisito de cientificidad consistente en contar con una tradición exitosa de resolución de problemas, del requisito de contar con un paradigma dominante. Como ha señalado Gómez Rodríguez (1997: 145) la búsqueda del consenso dentro de un paradigma no implica necesariamente la unidad paradigmática del tipo que han experimentado las ciencias naturales. Puede considerarse que las ciencias sociales son intrínsecamente multiparadigmáticas, y que si bien pueden tener paradigmas relativamente dominantes, seguramente nunca alcanzarán una homogeneidad paradigmática similar a la de las ciencias naturales, (Friedrichs, 1970; Ritzer, 1980), e introducir la interrogante respecto a si los paradigmas que componen cada disciplina social son capaces o no de organizar el trabajo comunitario conforme a los patrones de la ciencia madura. En cuanto a las ciencias de la información, puede pensarse que constituyen un campo más bien multiparadigmático, y que la convivencia de varios paradigmas no es un indicador de pre-cientificidad, (Capurro, 2007: 13). Puede aceptarse esta lectura de Kuhn, o puede considerarse que la madurez implica unidad paradigmática, por lo que sólo pueden considerarse ciencias maduras las disciplinas sociales que cuenten con un paradigma dominante. Pero en uno u otro caso lo que define la madurez es la conformación de una tradición exitosa y normalizada en cuanto a la resolución de problemas, más allá de si esta tradición se conforma en el marco de un paradigma dominante o en una situación de diversidad paradigmática. Esto implica que la consideración de la capacidad de resolver problemas como condición de cientificidad es independiente de si se defiende o se niega la unidad paradigmática como objetivo histórico de las ciencias sociales.

Otro contraste definido que aparece en La estructura de las revoluciones científicas remite a que los científicos naturales no están obligados a darles cuenta a los profanos de los términos en los que resuelven los problemas de su campo, ni a abordar problemas en razón de su urgencia social. Esto define el aislamiento de las comunidades científicas respecto del contexto, y permite dar cuenta de la rapidez y la sistematicidad con que los científicos naturales resuelven los rompecabezas que eligen, en cuanto la elección se 
orienta hacia aquellos problemas que el científico está convencido de poder resolver en función de las herramientas que le provee su paradigma, (Kuhn, 1962: 254). De aquí que en ciencias sociales siempre esté latente la riesgosa posibilidad de que los científicos desperdicien su energía resolviendo problemas que no pueden solucionarse con las herramientas que proporciona el paradigma, (idem: 71). A este respecto Sander (1989: 32-33), presenta una revisión del desarrollo de la teoría de la clasificación a finales del siglo XIX y principios del XX, mostrando la influencia del contexto sociocultural tanto en los problemas abordados como en las soluciones esbozadas por los teóricos de la clasificación. Del mismo modo Linares Columbié (2004), analiza las coyunturas sociales que en los siglos XIX y XX dieron lugar al surgimiento de la bibliotecología y la ciencia de la información respectivamente.

La argumentación de Kuhn sobre el aislamiento de los científicos naturales resulta bastante sugestiva por diferentes razones. En primer término, si el aislamiento es una de las condiciones de la madurez disciplinar y por lo tanto del progreso, las distancias entre las ciencias sociales y las naturales parecen difíciles de zanjar, en cuanto no resulta fácil concebir a las ciencias sociales resolviendo problemas que no estén directamente ligados con las necesidades sociales derivadas del contexto. Por otro lado, la imagen de las ciencias naturales que ofrece Kuhn resulta sumamente discutible desde el punto de vista histórico, en cuanto las orientaciones de investigación de los científicos naturales han estado, sobre todo en el siglo XX, mucho más influidas por las necesidades del contexto social de lo que Kuhn parecía estar dispuesto a aceptar. El impulso que toman las investigaciones sobre la fisión nuclear luego de haber sido puesto en marcha el Proyecto Manhattan seguramente sea un caso paradigmático, pero no el único correspondiente a una correlación cada vez más estrecha entre ciencia y desarrollo tecnológico. Pero admitiendo que para Kuhn el aislamiento es una condición para la madurez científica, es necesario insistir en que no es una condición determinante, en cuanto reconoce en pasajes de La estructura de las revoluciones científicas la influencia del contexto social en algunos cambios revolucionarios, (Kuhn, 1962: 15, 117), por lo que la consolidación de una tradición normal de resolución de problemas tendría un cierta independencia del nivel de aislamiento social presentado por cada disciplina.

\section{Hermenéutica y ciencia}

Kuhn retomó el problema del status de las ciencias sociales en un trabajo de 1989, "Las ciencias naturales y las humanas", en tren de debatir la caracterización 
de las ciencias sociales ofrecida por Charles Taylor (1985). Desde el punto de partida Kuhn se plantea si las diferencias entre las disciplinas sociales y las naturales son de fondo o si responden a diferencias en el desarrollo histórico de ambos grupos disciplinares, (Kuhn, 1989: 263). Siguiendo la terminología de Taylor, señala en principio que las ciencias naturales no son empresas hermenéuticas, en cuanto la investigación normal que las define no está orientada a la generación de nuevas interpretaciones, sino a la resolución de problemas definidos en la interna de un paradigma. En este sentido, cuando en las ciencias naturales han ocurrido revoluciones, quienes dieron lugar a los cambios no lo hicieron deliberadamente, sino siendo inconcientes del impacto futuro de su trabajo (idem: 264).

Las ciencias sociales por el contrario, son actividades fundamentalmente hermenéuticas, en cuanto la generación de nuevas interpretaciones alternativas a las ya existentes es "el propósito reconocido del juego", (idem: 264). Kuhn detectó en este carácter de las ciencias sociales un indicador explicativo de su inmadurez histórica respecto de las ciencias naturales, pero sin tomarlo como un rasgo estructural irreversible, (idem: 264$).{ }^{10}$ Kuhn parecía manejar la convicción — presentada de un modo no muy enfático- de que existen suficientes elementos históricos para inclinarse por la posibilidad de que las ciencias sociales alcancen en el futuro un estado de investigación normal:

Mucho de lo que se dice normalmente para argumentar la imposibilidad de llevar a cabo una investigación de resolución de rompecabezas en las ciencias humanas se decía hace dos siglos para vetar la posibilidad de una ciencia de la química, y se repitió un siglo más tarde para mostrar la imposibilidad de una ciencia de los seres vivos. Muy probablemente la transición que estoy indicando ya está en marcha en algunas especialidades actuales dentro de las ciencias humanas, (idem: 264).

Sin embargo, para contrarrestar esta perspectiva, introduce un curioso argumento acerca de la inestabilidad de los fenómenos estudiados por las ciencias sociales. En las ciencias naturales — señala- el fenómeno en estudio permanece estable a lo largo de la transición revolucionaria entre paradigmas, como permanecieron estables los cielos durante la revolución copernicana. Pero en las ciencias sociales, y en el estudio de fenómenos políticos y sociales, un nivel de estabilidad similar no parece fácil de asegurar, (idem: 264).

10 En La estructura de las revoluciones cientificas afirma: "En ciertas partes de la biología -por ejemplo, el estudio de la herencia- los primeros paradigmas universalmente aceptados son todavía más recientes; y queda todavía en pie la pregunta de qué partes de las ciencias sociales han adquirido ya tales paradigmas", (Kuhn, 1962: 40). 
Es significativo señalar que en su argumentación Kuhn está discutiendo dos problemas al mismo tiempo: por un lado si las diferencias entre las ciencias sociales y las naturales son de orden histórico o de orden estructural, y por otro, en caso de que sean de orden histórico, cuáles son las condiciones bajo las que las disciplinas sociales podrían alcanzar la madurez de las naturales. Estas dos cuestiones tienen no obstante un punto de convergencia, que radica en la importancia asignada por Kuhn a la capacidad de una disciplina para resolver problemas dentro de una tradición normalizada, (Kuhn, 1963). Cabe pensar que desde la perspectiva kuhniana, si las diferencias entre las disciplinas sociales y las naturales son de orden estructural, esto responde a que las ciencias sociales no pueden desarrollar elementos que permitan la resolución normalizada de problemas; y si por el contrario las diferencias son de orden histórico —y por tanto superables- la brecha entre ambos grupos de disciplinas se diluirá en la medida en que las ciencias sociales puedan desarrollar estos elementos.

En ambos casos, lo que hace la cientificidad de un grupo disciplinar es su capacidad de resolver problemas, ya que los intentos exitosos terminan de modo más o menos paulatino delimitando campos disciplinares. En estos términos, parece no tener demasiado contenido la posibilidad de que el advenimiento de un paradigma se produzca mediante una tentativa de fundamentación filosófica del trabajo desarrollado en la interna de una disciplina, o de su legitimación a través de un criterio de cientificidad. Las tareas de elucidación de los fundamentos filosóficos de una disciplina, y de defensa de su condición científica, se encuentran en un nivel diferente de los mecanismos de resolución de problemas que Kuhn consideraba definitorios de la cientificidad de un campo. Para clarificar esta distinción, Kuhn identificó cuatro condiciones para considerar que una disciplina ha alcanzado la madurez en su desarrollo histórico: 1. la disciplina debe producir predicciones concretas para algún campo de la naturaleza; 2 . debe alcanzar cierto éxito predictivo en algunos de los fenómenos contenidos en $1 ; 3$. debe contar con una teoría que a) explique el alcance de las predicciones, tanto en su éxito como en su fracaso, y b) indique medios para mejorarlas; finalmente 4. la mejora de las técnicas predictivas debe ser asumida por la comunidad como una tarea prioritaria, (Kuhn, 1970: 169).

La fundamentación filosófica de una disciplina, la introducción de una definición de ciencia o de un criterio de demarcación que la legitime, son actividades que pueden localizarse dentro de la tercera característica consignada por Kuhn, pero sólo de un modo parcial. Esto en cuanto se trata de estrategias que pueden ser útiles para dar cuenta del éxito y las limitaciones predictivas de una disciplina, o indicar cómo mejorar su capacidad de 
predicción, pero que no necesariamente cumplen este papel, ni de por sí lo hacen con éxito. Es posible introducir en defensa de una disciplina un criterio de cientificidad o una definición de ciencia no relacionados con la predictibilidad o la capacidad de resolver problemas, por lo que el desarrollo de estas estrategias no asegura el cumplimiento de las funciones 3.a y 3.b introducidas por Kuhn, si bien tampoco lo impide. En cuanto a las otras tres características, éstas remiten a la articulación de la disciplina con el campo de fenómenos que definen su objeto, y es en ese plano donde variables como la capacidad predictiva y los problemas resueltos definen la madurez disciplinar.

En estos términos, la negativa a tomar el esclarecimiento de los fundamentos filosóficos de una disciplina como condición para su cientificidad no supone negar el componente metafísico presente de forma ineludible en el trabajo científico, sólo supone establecer que el trabajo especulativo sobre dicho componente no es la condición fundamental para defender las pretensiones de cientificidad de una disciplina.

\section{Conclusión}

En virtud de lo que se ha argumentado hasta aquí, cabe pensar hasta qué punto en el debate sobre el status científico de las ciencias de la información no ocurre un traslado categorial en función del cual predomina la acepción de paradigma como conjunto de compromisos ontológicos por sobre la acepción referente a problemas resueltos con éxito. Desde este punto de vista, cuando en la literatura se emplea la expresión paradigmas en ciencias de la información, el énfasis parece estar puesto más en las estructuras conceptuales de las teorías que conforman el campo que en los casos exitosos de resolución de problemas.

Esto no implica que desde una perspectiva kuhniana queden deslegitimados los debates acerca de la naturaleza de una disciplina o los intentos de elucidar sus fundamentos. Pero sí supone esclarecer el alcance y la dimensión de estos debates, que constituyen antes un indicador de la falta de consolidación paradigmática en un campo, que una herramienta para lograr dicha consolidación. Esto en cuanto a las disciplinas que han alcanzado status paradigmático no lo han logrado a través de este tipo de estrategias, sino a través de la resolución exitosa de alguna serie relevante de problemas. No obstante, teniendo en cuenta el desarrollo histórico de las ciencias de la información y de las ciencias sociales en general, cabe pensar que los debates acerca de los fundamentos y la naturaleza de la disciplina son una consecuencia estructural de 
la ausencia de paradigmas consolidados, y seguirán proliferando mientras el campo no se consolide. ${ }^{11}$ Cumplen por tanto una función relevante a efectos de clarificar las bases de la disciplina y de su reconstrucción histórica, y en este sentido contribuyen a la consolidación paradigmática. Pero el alcance de esta contribución es acotado, en cuanto la consolidación disciplinar responde mucho más a la resolución exitosa de problemas que a este tipo de estrategias de fundamentación.

\section{Bibliografía}

Ávila Araújo. C. A. (2003), "A ciencia da informação como ciencia social”, en Ciencia da informação, 32 (3), 21-27.

Barnes, B., (1928), T. S. Kubn y las ciencias sociales, México, Fondo de Cultura Económica.

Borko, H. (1968), "Information Science. What it is?", en American Docummentation, 19 (1), 3-5.

Busha, C. H.; Harter, S. P. (1980), Research Methods in Librarianship: technique and interpretation, New York, Academic Press.

Capurro, R. (2007), “Epistemología y ciencia de la información”, en Enlace, 4 (1), 11-29.

Cordero Ulate, A. (2008), El paradigma inconcluso. Kubn y la sociología en América Latina, Guatemala, FLACSO.

de Souza, S. (1996), "Información: utopía y realidad de la Bibliotecología”, en Investigación Bibliotecológica, 10 (21), 14-17.

Eckberg, D.; Hill, L. (1980), “The Paradigm Concept and Sociology: A Critical Review”, en Gutting, G. (ed.), Paradigms and Revolutions. Appraisals and Aplications of Thomas Kubn's Philosopby of Science, Notre Dame, University of Notre Dame Press.

Friedrichs, R. (1979), A Sociology of Sociology, New York, The Free Press.

Fuller, S. (2000), Thomas Kubn. A philosophical History for our times, Chicago, The University of Chicago Press.

Gadamer, H. G. (1965), Verdad y método, Salamanca, Sígueme, 1984.

Gómez Rodríguez, A. (1997), "Kuhn y las ciencias sociales”, en Endoxa, 9, 139-166.

11 Puede objetarse a esto que el campo de las ciencias de la información ya está consolidado; pero esta objeción sólo es posible bajo un concepto de consolidación que no sea el de Kuhn. Para Kuhn un campo está consolidado cuando da forma a paradigmas articulados que sustentan la investigación normal destinada a la resolución de enigmas; y dentro de las ciencias sociales, sólo algunos sectores de la economía y la psicología le parecían merecedores de semejante consideración, (Kuhn, 1989: 264). Bajo otro marco epistemológico puede ser lícito y aceptable considerar que el campo de las ciencias de la información está consolidado - y plantear el debate en esos términos-, pero la perspectiva de Kuhn no ofrece elementos que sustenten dicha afirmación. 
Heisenberg, W. (1971), Más allá de la física, Madrid, BAC, 1974.

Isakovic, D. (1965), "La documentologie", en Revue Internationale de la Documentation, 32 (4), 152-153.

Kuhn, T. (1962), La estructura de las revoluciones científicas, México, FCE, 2004.

(1963), "The function of dogma in scientific research", en Crombie, A. C. (comp.), Scientific Change: Historical Studies in the Intellectual, Social and Technical Conditions for Scientific Discovery and Technical Invention, from Antiquity to Present, London, Heinemann, pp. 347-369.

(1970), "Consideraciones en torno a mis críticos", en El camino desde la Estructura, Barcelona, Paidós, 2002, 151-209.

(1974), “Algo más sobre paradigmas", en La tensión esencial, México, Fondo de Cultura Económica, 1993.

(1989), "Las ciencias naturales y las sociales", en El camino desde la Estructura, Barcelona, Paidós, 2002, pp. 257-265.

Lakatos, I. (1970), "La falsación y la metodología de los programas de investigación científica”, en La metodología de los programas de investigación científica. Escritos filosóficos 1, Madrid, Alianza, 2007.

Laudan, L. (1977), El progreso y sus problemas, Madrid, Encuentro, 1986.

Linares Columbié, C. (2004), "Bibliotecología y Ciencia de la Información: ¿subordinación, exclusión o inclusión?”, en Acimed, 12 (3).

López Yepes, J. (1978), Teoría de la documentación, Pamplona, Eunsa. Morales López, V. (1998), "Acercamiento al concepto de metodología bibliotecológica”, en Investigación Bibliotecológica, 12 (25), 33-50.

Otero, E. (2004), "La distinción kuhniana entre tipos de ciencia y la inconsistencia fundacional de los estudios sociales de la ciencia”, en Ciencias Sociales on line, I (1), 1-7.

Popper, K. (1934), La lógica de la investigación cientifica, Madrid, Tecnos, 1997.

Puntel Mostafa, S. (1996), "Enfoques paradigmaticos da bibliotecologia; unidade na diversidad ou diversidad na unidad", en Investigación Bibliotecológica, 10 (21), 18-21.

Rendón Rojas, M. A. (1996), "Debate abierto sobre la epistemología de la Bibliotecología”, en Investigación Bibliotecológica, 10 (21), 34 36.

(2000), "La ciencia bibliotecológica y de la información ¿tradición o innovación en su paradigma científico”, en Investigación Bibliotecológica, 14 (28), 34-52.

Ritzer, G. (1975), "Sociology: a multiple paradigm science", en The American Sociologist, 10 (3), 156-167.

(1980), Sociology: a multiple paradigm science, Boston, Allyn and Bacon. 
Sander, S. (1989), "Elementos histórico-teóricos para la indagación de la estructura teórica de la Bibliotecología”, en Investigación Bibliotecológica, 3 (6), 31-37.

Shera, J. (1972), The Foundations of Education For Librarianship. New York, Beckerand Hayes.

Taylor, C. (1985), "La interpretación y las ciencias del hombre”, en La libertad de los modernos, Buenos Aires, Amorrortu, 2005.

Taylor, R. (1963), “The Information Sciences”, en Library Journal, 4161-4163.

Velazco Gómez, A. (2000), Tradiciones naturalistas y hermenéuticas en la filosofía de las ciencias sociales, México, UNAM. 